\title{
Diagnostic utility of measuring lactate dehydrogenase levels and its isoenzyme activities for the evaluation of malignancy in feline pleural effusion and ascitic fluid
}

\section{Eiji Naito}

Yuki Animal Hospital

\section{Roka Shimada}

Yuki Animal Hospital

Masashi YUKI ( $\square$ yuki-masashi@mvf.biglobe.ne.jp)

Yuki Animal Hospital https://orcid.org/0000-0002-0803-0760

\section{Short Report}

Keywords: ascitic fluid, lactate dehydrogenase, lactate dehydrogenase isoenzymes, malignancy, pleural effusion

Posted Date: November 11th, 2021

DOl: https://doi.org/10.21203/rs.3.rs-984351/v1

License: (c) (1) This work is licensed under a Creative Commons Attribution 4.0 International License.

Read Full License 


\section{Abstract}

Lactate dehydrogenase (LDH) isoenzymes may be useful in the differential diagnosis of pleural effusion (PE) and ascitic fluid (AF) etiologies in cats since tissue damage induces their release, thereby changing the pattern of their activity. The present study aimed to determine the diagnostic utility of measuring LDH levels and its isoenzyme activities in PE or AF in cats with malignancy. We measured LDH levels and its isoenzyme activities in serum, $\mathrm{PE}$, and $\mathrm{AF}$ in 29 cats and compared the results between malignant, infectious diseases, and non-malignant, non-infectious diseases. Receiver operating characteristic (ROC) analysis was performed to assess the accuracy of the tests in diagnosing feline malignancy. In PE or AF, significant differences were found in LDH levels and LDH isoenzymes activities among the three groups. The combination of LDH level and LDH-1 activity in PE or AF had the highest area under the ROC (AUC) value of 0.874 for discriminating malignant effusion from non-malignant effusion. The sensitivity and specificity of using the combination of LDH level (cutoff: $<2,269 \mathrm{U} / \mathrm{L}$ ) and LDH-1 activity (cutoff: $<4.8 \%$ ) in $\mathrm{PE}$ or $\mathrm{AF}$ for predicting malignancy with the highest AUC value were $94.4 \%$ and $72.7 \%$, respectively. Our results suggest that the combination of $\mathrm{LDH}$ level and $\mathrm{LDH}-1$ activity in $\mathrm{PE}$ or $\mathrm{AF}$ can potentially diagnose for malignancy. Considering that LDH isoenzymes can be measured inexpensively and easily, LDH tests can be readily accommodated in veterinary clinical practice.

\section{Introduction}

Pleural effusion (PE) and ascitic fluid (AF) can present interesting diagnostic challenges in cats. Differential diagnoses related to PE and AF in cats could include a variety of diseases (Ettinger et al. 2017). However, the classification of PE and AF into malignant or non-malignant remains challenging, despite using various tests.

In humans, lactate dehydrogenase (LDH), which plays a role in the glycolytic way, has been the focus in the differential diagnosis of various diseases because of their release from cells after cell damage or death (Dawson et al. 1964). The distributions of LDH five isoenzymes differ in various tissues (Lott and Stang 1980; Wroblewski and Gregory 1961). However, the diagnostic values of serum LDH level and LDH isoenzyme patterns are still controversial (Dawson et al. 1964; Wroblewski and Gregory 1961). In contrast, LDH isoenzyme activities in PE and AF are useful biomarkers for distinguishing neoplastic and non-neoplastic diseases (Cobben et al. 1997; Lossos et al. 1999; Paavonen et al. 1991; Sevinc et al. 2005). Interestingly, LDH-1 activity, which works efficiently under aerobic conditions, is significantly lower in neoplastic diseases than in non-neoplastic diseases, whereas LDH-4 level, which works efficiently under anaerobic conditions, is significantly higher in neoplastic diseases than in non-neoplastic diseases (Paavonen et al. 1991; Sevinc et al. 2005).

In veterinary medicine, serum LDH level in cats with lymphoma are a potential negative prognostic indicator of survival (Hadden et al. 2008). Also, LDH level in PE in cats with feline infectious peritonitis (FIP) and pyothorax were increase (Zoia et al. 2009). However, few reports have been made regarding $\mathrm{LDH}$ isoenzyme analyses in feline PE and AF. 
The present study evaluated LDH level and LDH isoenzyme activities in serum, PE, and AF in cats with neoplastic and non-neoplastic diseases. We hypothesized that LDH level and anaerobic LDH isoenzyme activities in PE and AF with neoplastic and infectious diseases would be higher than that with other diseases.

\section{Materials And Methods}

This retrospective cross-sectional study analyzed cats in which PE or AF was newly detected at the Yuki Animal Hospital from December 2014 to December 2018. These cats were divided into 2 groups based on diagnosis. The malignant group included cats with tumors that were diagnosed by the detection of tumor cells by fine needle aspiration, clonality analysis, or biopsy. The non-malignant group included cats with diseases that cause PE and AF without tumors. The non-malignant group included two subgroups: nonmalignant group 1: non-infectious diseases such as idiopathic chylothorax, congestive heart failure (CHF), and liver disease; and non-malignant group 2: infectious diseases such as FIP, pyothorax, and bacterial peritonitis. CHF was confirmed by cardiac ultrasonography. Liver disease was diagnosed by histopathological examination of the liver. FIP was diagnosed when supportive history, signalment, and clinical and clinicopathological data were present and no other causes of PE and AF could be identified (Zoia et al. 2009). Pyothorax and bacterial peritonitis were diagnosed by positive bacterial culture. The exclusion criteria of this study were cats with an incomplete diagnosis and cats with co-existing diseases that cause PE and AF. All serum, PE and AF samples were centrifuged and separated within $30 \mathrm{~min}$. All the samples were stored at $4^{\circ} \mathrm{C}$ during the experiment. The LDH level and LDH isoenzyme activities were measured in all samples (LSI Medience Co., Tokyo, Japan). The results of each laboratory test and the ages of the cats were compared among the malignant group, non-malignant group 1, and non-malignant group 2 using Kruskal-Wallis test. Post hoc comparisons were conducted using Mann-Whitney $U$ test with Bonferroni correction. The accuracy of the tests between the malignant and non-malignant groups was analyzed by receiver operating characteristic (ROC) analysis. The area under the ROC curve (AUC) and sensitivity and specificity with 95\% confidence intervals (CI) were calculated. Logistic regression was performed to evaluate the diagnostic accuracy of malignancy by the combination of the LDH level and LDH isoenzyme activity used for ROC analysis. A P value of $<0.05$ was considered significant. Statistical analyses were performed using Easy R software (Kanda 2013).

\section{Results}

Forty-nine cats were newly diagnosed with PE or AF. The malignant group included 11 cats, 5 with PE and 6 with AF. The cats with PE were diagnosed with the following diseases: 3 with lung carcinoma, 1 with mediastinal lymphoma, and 1 with extranodal lymphoma. The cats with AF were diagnosed as follows: 3 with alimentary lymphoma, 1 with renal lymphoma, 1 with intestinal carcinoma, and 1 with hepatocellular carcinoma. Non-malignant group 1 included 12 cats, 10 with PE and 2 with AF. The cats with PE were diagnosed as follows: 7 with hypertrophic cardiomyopathy, 1 with dilated cardiomyopathy, 1 with restrictive cardiomyopathy, and 1 with unclassified cardiomyopathy. The cats with AF were diagnosed as 
follows: 1 with hypertrophic cardiomyopathy by ultrasonography and 1 with cholangitis and hepatitis by histopathological liver examination. Non-malignant group 2 included 6 cats, 2 with PE and 4 with AF. Two cats with PE were diagnosed with FIP. Three cats with AF were diagnosed with FIP, and 1 cat was diagnosed with bacterial peritonitis. Twenty cats were excluded.

The cat breeds included 25 crossbreeds, two Scottish Folds, and two Ragdolls. The malignant group consisted of 6 males ( 2 castrated) and 5 females ( 3 spayed). The median age was 12.2 years (range, 317.4 years). Non-malignant group 1 consisted of 6 males ( 4 castrated) and 6 females ( 4 spayed). The median age was 16 years (range, 6.8-21.4. years). Non-malignant group 2 consisted of 5 males (2 castrated) and 1 intact female. The median age was 3.8 years (range, $0.4-17.0$ years).

Data of LDH and LDH isoenzymes were shown in Table and Figure 1. In serum, there were no significant differences in LDH level and LDH isoenzymes activities among 3 groups. In PE and AF, the LDH level in the malignant group (median, $441 \mathrm{U} / \mathrm{L}$; interquartile range [IQR], 151-1,016 U/L) was significantly higher than that in the non-malignant group 1 (median, $65 \mathrm{U} / \mathrm{L} ; \mathrm{IQR}, 47-93 \mathrm{U} / \mathrm{L})(P=0.007)$, and lower than that in the non-malignant group 2 (median, 2,477 U/L; IQR, 2,284-2,928 U/L) $(P=0.015)$. The LDH-1 activity in the malignant group (median, 4.4\%; IQR, 3.5\%-6.0\%) was significantly lower than that in non-malignant group 1 (median, 29.6\%; IQR, 23.4\%-44.5\%) $(P<0.001)$, and higher than that in non-malignant group 2 (median, $2.0 \%$; IQR, $1.3 \%-2.7 \%)(P=0.031)$. The LDH-2 activity in non-malignant group 1 (median, $15.9 \%$; IQR, $12.0 \%-17.1 \%$ ) was significantly higher than that in non-malignant group 2 (median, 6.0\%; IQR, 4.5\%-7.0\%) ( $P=0.004)$. The LDH-3 activity in the malignant group (median, 20.4\%; IQR, 20.1\%-25.0\%) was significantly lower than that in the non-malignant group 1 (median, $10.1 \%$; IQR, $9.5 \%-12.5 \%)(P=0.007)$. The LDH-4 activity in the malignant group (median, $31.8 \%$; IQR, $29.5 \%-34.7 \%$ ) was significantly lower than that in the non-malignant group 1 (median, $16.8 \%$; IQR, $12.9 \%-23.9 \%)(P=$ $0.002)$, and lower than that in the non-malignant group $2(37.6 \% ; 36.2 \%-39.4 \%)(P=0.015)$.

The results of the ROC curve analysis are shown in Table. The combination of LDH level and LDH-1 activity in PE or AF showed the highest AUC value for discriminating malignant effusion from nonmalignant effusion compared with other factors. The AUC of the combination of LDH level and LDH-1 activity in PE or AF was 0.874 (95\% Cl: $0.739-1.000)$, with a sensitivity and specificity $94.4 \%$ and $72.7 \%$, respectively (cutoff: LDH, $<2,269 \mathrm{U} / \mathrm{L} ; \mathrm{LDH}-1$ activity, $<4.8 \%$ ).

\section{Discussion}

The present study demonstrated the diagnostic utility of the LDH level and LDH isoenzyme activities in feline PE and AF. Especially, the combination of the LDH level and LDH-1 activity had the highest diagnostic potential in distinguishing malignancy from non-malignancy. In our study, neoplastic disease behaved similarly to infectious diseases in LDH level and LDH isoenzyme activities. Furthermore, infectious diseases tend to produce higher LDH level than other diseases, whereas infectious diseases tend to have lower LDH-1 activity than other diseases. Generally, cytological tests for malignant cells in PE and AF demonstrate high specificity (100\%) but low sensitivity (50\%) (Spangler et al. 2000). In the 
present study, all cats in the malignant group did not be diagnosed by cytological testing with PE and AF; therefore, LDH isoenzyme analyses have a potential of diagnostic biomarker regardless of cytological testing with PE and AF.

In the present study, there were no significant differences in serum LDH level and LDH isoenzyme activities among 3 groups. By contrast, the LDH level and LDH isoenzyme activities in PE and AF differed significantly among neoplastic, infectious diseases and non-malignant, non-infectious diseases. These differences in LDH levels and LDH isoenzyme activities may be caused by differences between body fluids and blood. Saint-Rémy et al. (1986) reported that the LDH level and LDH isoenzyme activities might be affected by the release of enzymes from inflammatory cells and erythrocytes present in body fluid. Paavonen et al. (1991) reported that visceral or parietal pleural cells are rich in LDH. For these reasons, Sevinc et al. (2005) deemed that LDH isoenzymes might be released from cells that infiltrate body fluids other than serum. Thus, the LDH level in feline body fluids may be higher than in serum, similar to that in humans, and the differences between body fluids and serum are easily observed.

LDH is present in essentially all organ systems and is released from cells only after cell damage or death (Drent et al. 1996; Glick et al. 1969). Elevated LDH levels in PE or AF were caused by activated, injured, or dead leukocytes, tumor cells, or mesothelial cells (Sevinc et al. 2005; Washizu et al. 2002). The LDH level depends on the number of cells, such as leukocytes, tumor cells, or mesothelial cells in PE and $A F$, because $L D H$ is released from cells. Moreover, $L D H$ is also released from dead cells, regardless of the number of cells in PE and AF. Therefore, significant differences were observed among 3 groups in the present study due to the difference in the origin of LDH.

This study demonstrated that LDH-1 activity in PE or AF increased in cats with CHF and liver disease. In humans, $\mathrm{CHF}$ increases the percentile of $\mathrm{LDH}-1$ activity in $\mathrm{PE}$ and $\mathrm{AF}$ due to aerobic conditions in heart tissue (Firth et al. 1995; Cobben et al. 1997; Sevinc et al. 2005). Increasing in LDH-1 activity in AF was also observed in patients with chronic liver disease (Sevinc et al. 2005). These findings were reflected in the diagnoses of the cases in the present study. In addition, LDH-3 and LDH-4 activities increased in the $\mathrm{PE}$ or AF of cats with neoplastic and infectious diseases. Tumor cells need LDH to maintain and increase (Fantin et al. 2006). Hypoxic areas in malignancy have been shown to increase (Fantin et al. 2006). Human LDH-4 activity in PE and AF were found to be significantly higher in malignancy than in nonmalignancy (Cobben et al. 1997; Sevinc et al. 2005). These studies included patients with non-blood cancers. Whereas, the malignant group in the present study included 6/11 cats with lymphoma. Anaerobic LDH isoenzymes have been reported the most abundant isoenzyme in lymphoid tissue (Dumontet et al. 1999). Moreover, leukocytes have more anaerobic LDH isoenzymes in cats (Washizu et al. 2002). These results suggested that the distribution of LDH-3 and LDH-4 in neoplastic and infectious group in cats were increase.

This study investigated PE and AF together. Generally, LDH isoenzymes content vary across different organs. However, in our research, no significant differences in LDH level and LDH isoenzyme activities were found between PE and AF in each of the malignant and non-malignant groups (data not 
shown). Therefore, we included cats with both PE and AF. The malignant group included various neoplastic diseases. The age, weight, and breed in each group were not consistent. Given that only examined cats were selected, our findings may not be reflective of all cats with malignancy.

In conclusion, the present study suggests that the combination of LDH level and LDH isoenzyme activities in PE or AF are potential factors for diagnosing malignancy.

\section{Declarations}

\section{Acknowledgments}

The authors wish to thank all staff at the Yuki Animal Hospital for their support. The authors also wish to thank Mr. Hosoda (LSI Medience Corp.) for his technical assistance in the acquisition of LDH data.

\section{Authors' Contributions}

EN designed the study. EN, RS, and MY participated in sample collection. EN and RS collected and analyzed the data and drafted the manuscript. All authors read and approved the final manuscript.

Funding: The authors received no financial support for the research, authorship, and/or publication of this article.

Conflict of Interest: The test for LDH level and LDH isoenzyme activity measurements was provided by LSI Medience Corp. The study plan, data analyses, data interpretation, and decision to publish the results were not influenced by the funders.

Data Availability: The data that support the findings of this study are available from the corresponding author (M.Y.) upon reasonable request.

Ethical Approval: This work involved the use of non-experimental owned cats only. In Japan, there is no ethics committee available for private-practice animal hospitals. Nevertheless, this research was conducted according to the ethical codes of the Japan Veterinary Medical Association.

Consent to participate: The samples obtained in this study were used after obtaining consent from each cat owner.

Consent for Publication: Not applicable.

\section{References}

Cobben NA, van Belle AF, Pennings HJ, Mulder PG, van Dieijen-Visser MP, Wouters EF, Drent M (1997) Diagnostic value of lactate dehydrogenase isoenzyme pattern in pleural effusions. Eur $\mathrm{J}$ Clin Chem Clin Biochem 35:523-528. https://doi.org/10.1515/cclm.1997.35.7.523 
Dawson DM, Goodfriend TL, Kaplan NO (1964) Lactic dehydrogenases: functions of the two types rates of synthesis of the two major forms can be correlated with metabolic differentiation. Science 143:929933. https://doi.org/10.1126/science.143.3609.929

Drent M, Cobben NA, Henderson RF, Wouters EF, van Dieijen-Visser M (1996) Usefulness of lactate dehydrogenase and its isoenzymes as indicators of lung damage or inflammation. Eur Respir J 9:17361742. https://doi.org/10.1183/09031936.96.09081736

Dumontet C, Drai J, Bienvenu J, Berard EN, Thieblemont C, Bouafia F, Bayle F, Moullet I, Salles G, Coiffier B (1999) Profiles and prognostic values of LDH isoenzymes in patients with non-Hodgkin's lymphoma. Leukemia 13:811-817. https://doi.org/10.1038/sj.leu.2401417

Ettinger SJ, Feldman EC, Cote E (2017) Textbook of veterinary internal medicine. 8th edn. Saunders Elsevier, St. Louis, pp 439-448.

Fantin VR, St-Pierre J, Leder P (2006) Attenuation of LDH-A expression uncovers a link between glycolysis, mitochondrial physiology, and tumor maintenance. Cancer Cell 9:425-434. https://doi.org/10.1016/j.ccr.2006.04.023

Firth JD, Ebert BL, Ratcliffe PJ (1995) Hypoxic regulation of lactate dehydrogenase A. Interaction between hypoxia-inducible factor 1 and cAMP response elements. J Biol Chem 270:21021-21027. https://doi.org/10.1074/jbc.270.36.21021

Glick JH (1969) Serum lactate dehydrogenase isoenzyme and total lactate dehydrogenase values in health and disease, and clinical evaluation of these tests by means of discriminant analysis. Am J Clin Pathol 52:320-328. https://doi.org/10.1093/ajcp/52.3.320

Hadden AG, Cotter SM, Rand W, Moore AS, Davis RM, Morrissey P (2008) Efficacy and toxicosis of VELCAP-C treatment of lymphoma in cats. J Vet Intern Med 22:153-157. https://doi.org/10.1111/j.19391676.2007.0031.x

Kanda Y (2013) Investigation of the freely available easy-to-use software 'EZR' for medical statistics. Bone Marrow Transplant 48:452-458. https://doi.org/10.1038/bmt.2012.244

Lossos IS, Intrator O, Berkman N, Breuer R (1999) Lactate dehydrogenase isoenzyme analysis for the diagnosis of pleural effusion in haemato-oncological patients. Respir Med 93:338-341. https://doi.org/10.1016/s0954-6111(99)90315-3

Lott JA, Stang JM (1980) Serum enzymes and isoenzymes in the diagnosis and differential diagnosis of myocardial ischemia and necrosis. Clin Chem 26:1241-1250.

Paavonen T, Liippo K, Aronen H, Kiistala U (1991) Lactate dehydrogenase, creatine kinase, and their isoenzymes in pleural effusions. Clin Chem 37:1909-1912. 
Saint-Rémy P, Buret J, Radermecker M (1986) Significance of lactate dehydrogenases in pleural effusions. Rev Pneumol Clin 42:74-81.

Sevinc A, Sari R, Fadillioglu E (2005) The utility of lactate dehydrogenase isoenzyme pattern in the diagnostic evaluation of malignant and nonmalignant ascites. J Natl Med Assoc 97:79-84.

Spangler EA, Rogers KS, Thomas JS, Pustejovsky D, Boyd SL, Shippen DE (2000) Telomerase enzyme activity as a diagnostic tool to distinguish effusions of malignant and benign origin. J Vet Intern Med 14:146-150. https://doi.org/10.1892/0891-6640(2000)0142.3.co;2

Washizu T, Nakamura M, Izawa N, Suzuki E, Tsuruno S, Washizu M, Nakajo S, Arai T (2002) The activity ratio of the cytosolic MDH/LDH and the isoenzyme pattern of LDH in the peripheral leukocytes of dogs, cats and rabbits. Vet Res Commun 26:341-346. https://doi.org/10.1023/a:1016278409138

Wroblewski F, Gregory KF (1961) Lactic dehydrogenase isozymes and their distribution in normal tissues and plasma and in disease states. Ann N Y Acad Sci 94:912-932. https://doi.org/10.1111/j.17496632.1961.tb35584.x

Zoia A, Slater LA, Heller J, Connolly DJ, Church DB (2009) A new approach to pleural effusion in cats: markers for distinguishing transudates from exudates. J Feline Med Surg 11:847-855. https://doi.org/10.1016/j.jfms.2009.04.005

\section{Tables}

Due to technical limitations, table 1 and 2 is only available as a download in the Supplemental Files section.

\section{Figures}



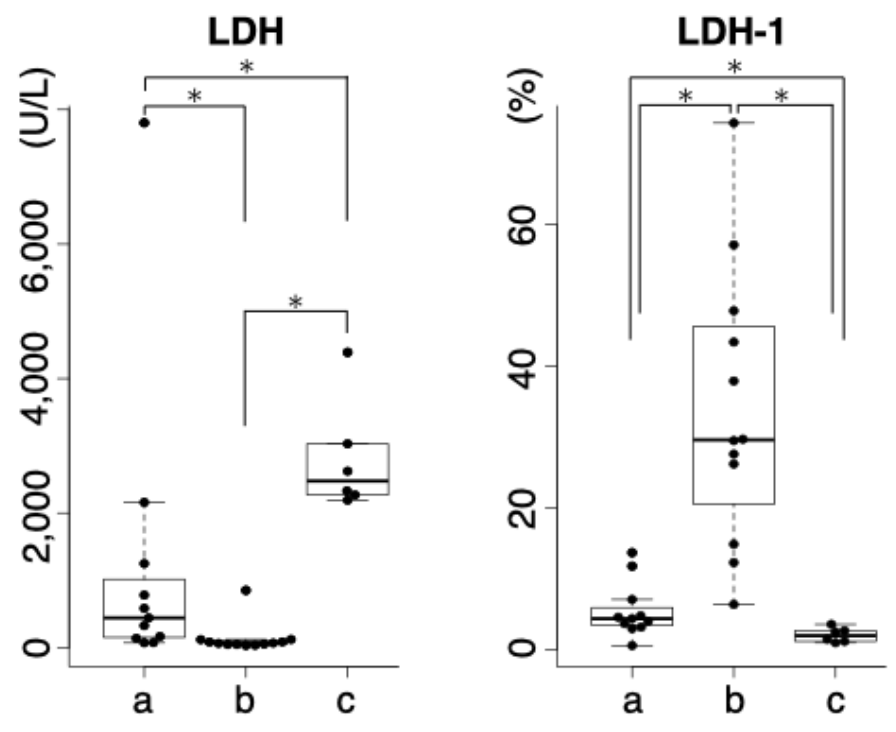

LDH-2

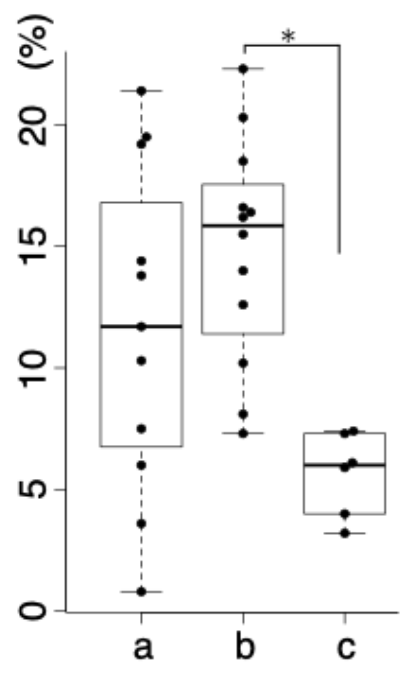

LDH-3

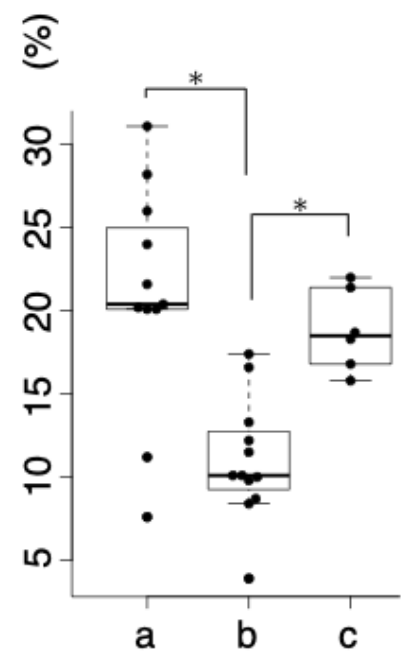

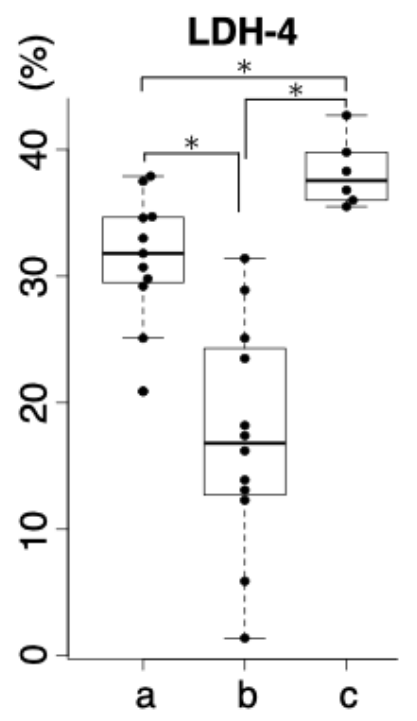

LDH-5

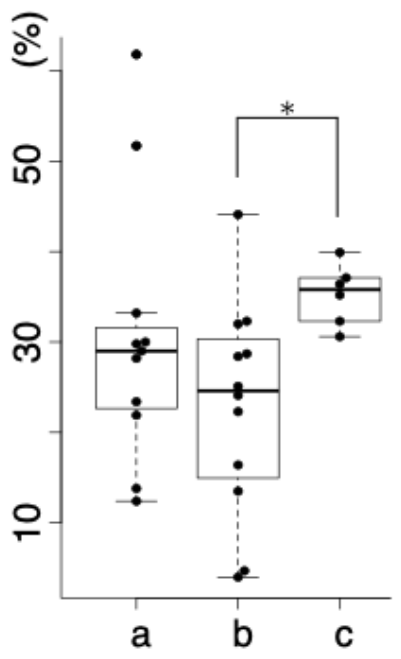

a: Malignant group

b: Non-malignant group 1

c: Non-malignant group 2

\section{Figure 1}

Comparison of LDH isoenzyme activities in PE or AF between the malignant and non-malignant groups Box plots show the median, range, and 25th and 75th quartiles within the groups. LDH level, LDH-3, and LDH-4 activities were significantly higher in the malignant group than in non-malignant group $1(\mathrm{P}=$ $0.007,0.007$, and 0.002 , respectively). LDH-1 activity was significantly lower in the malignant group than in non-malignant group $1(\mathrm{P}<0.001)$. LDH-1 activity was significantly lower in the malignant group than in non-malignant group $2(P=0.031)$. LDH level and LDH-4 activity were lower in the malignant group than in non-malignant group $2(P=0.015$, respectively). Statistical analyses were performed using the Kruskal-Wallis test. Post hoc comparisons were conducted using the Mann-Whitney $U$ test with Bonferroni correction. *P< $0.05 ; \mathrm{LDH}$, lactate dehydrogenase; $\mathrm{PE}$, pleural effusion; $\mathrm{AF}$, ascitic fluid.

\section{Supplementary Files}

This is a list of supplementary files associated with this preprint. Click to download. 
- CopyofLDHTable120211105.xIsx

- CopyofLDHTable220211105.xlsx 